\title{
Savings on Transmit Power through Designed Horizon Plane for LEO Satellite Ground Stations
}

\author{
Shkelzen Cakaj, Bexhet Kamo
}

\begin{abstract}
Low Earth Orbit (LEO) satellites are used for public networking and for scientific purposes. Established satellite ground stations can communicate with LEO satellites only when the satellite is in their visibility region. Each ground station is characterized by its own ideal horizon plane. Because of natural barriers that plane is modified to the designed one, defined by minimal elevation, in order to avoid natural obstacles. Designed horizon plane implementation implies also the power saving from the satellite to be transmitted. The major loss in communication between the LEO satellite and the ground station is the free space loss. Free space loss varies since the distance from the ground station to the satellite varies over time, usually compensated through variable satellite transmit power toward the downlink. The main novelty of the paper is the mathematical elaboration and confirmation that through the implementation of the designed horizon plane instead of an ideal one, the transmit EIRP (Equivalent Isotropic Radiated Power) from the satellite toward the ground station, could be saved and still permanently keeping the constant receiving performance at the ground station. The novelty is proved by simulation for different altitudes associated with power savings calculation for designed horizon plane implementation compared to the ideal one.
\end{abstract}

Keywords—LEO; satellite; EIRP; horizon.

\section{INTRODUCTION}

The demands for satellite services are increasing every day, especially in the field of providing broadband services [1]. Early designs were focused on making the system suitable for commercial operations, but new generations have to develop ways of maximizing the downlink data throughput related to broadband service requirements. [2]. The spacecraft orbit and mission goals will drive available locations for the ground stations. The important factors on ground station design are location, coverage, availability, user needs, cost and the components of the ground station architecture. The ground station design and architecture is the research subject by different worldwide institutions, among them to be emphasized the work by Toronto Institute for Aerospace Studies (UTIAS) related to nanosatellites and their respective ground stations [3]. Another institution is the Georgia Institute of Technology, Atlanta, which will support five small satellite missions within a two year frame (2017 to 2019).

Manuscript received April 3, 2018; revised July 21, 2018. Date of publication September 19, 2018. The associate editor Prof. Nikola Rožić has been coordinating the review of this manuscript and approved it for publication.

Authors are with the Faculty of Information Technology, Polytechnic University of Tirana, Albania (e-mails: \{ shcakaj, bkamo\}@fti.edu.al ).

Digital Object Identifier (DOI): 10.24138/jcomss.v14i3.534
The Georgia Institute of Technology will use a network of three different ground stations [4]. Satellite communication requirements depend on the satellite's mission nature. The ground station design should be in accordance with such requirements, also. In the both above cases, the designed ground station horizon plane and its impact on satellite's transmit power seems not to be deeply discussed.

Thus, the further author's elaboration is an approach toward designed ground station horizon determination and its impact on power savings. Nowadays, specifically for small and lightweight satellites (micro and nano) are applied flexible payload architectures with variable satellite EIRP and reconfigurable coverage in order to maximize satellite mission performance [5]. On-board processing models with reconfiguration and self-configuration are considered, also [6]. Variable satellite's EIRP is almost mandatory to keep receiving ground station performance for LEO satellites, because of variation in distance between the satellite and the ground station. Authors' interest is the satellite ground station performance. The idea is keeping the ground station performance constant, under different propagation and communication circumstances. LEO satellites are characterized by variable free space loss, since the range between the ground station and the satellite varies over time. Free space loss variation should be compensated by EIRP. Authors through their paper [7] presented the idea that such variation of the EIRP at the satellite to be transformed at the bandwidth tuning at the receiver of the satellite ground station, bringing the complexity from the satellite to the ground station. But, the continuous change of the bandwidth faces serious difficulties to be implemented and the bandwidth tuning may raise interference and regulatory issues.

This paper is another attempt and consideration related to the satellite's power to be transmitted toward the ground station. The paper approaches the EIRP savings at the satellite by keeping the constant receiving signal margin at the ground station, through the implementation of the designed horizon plane, instead of an ideal one. In order to obtain the constant downlink margin at the receiver (constant signal to noise ratio), all over the time, for analytical and simulation purposes, the typical LEO altitudes from $600 \mathrm{~km}$ to $1200 \mathrm{~km}$ are considered. For these altitudes, under different elevations, are simulated and calculated the EIRP savings. For simulation purposes the increment steps of $200 \mathrm{~km}$ for altitudes and of $5^{\circ}$ for elevation are applied. Behind the Introduction, by Section II the satellite ground station geometry is considered, followed by elaboration of the satellite ground station horizon and designed plane given at Section III. Section IV is dedicated to 
downlink link budget estimation. By Section V, the power savings simulation is presented, proving the power savings by designed horizon implementation compared to the ideal one. Finally, the paper is concluded by methodology of designed horizon plane determination, for an in advance defined power to be saved.

\section{SATELlite AND GROUND StATION GEOMETRY}

The location of the satellite ground station (could be any point on the Earth's surface) is usually given in terms of geographical coordinates defined by latitude and longitude. The main communication goal is to establish the link between the appropriate ground station and the satellite. In Figure 1.a) is presented the case where the GS (Ground station) has no communication with the satellite being still out of the LEO coverage zone and in Figure 1.b) is presented the case where the GS (Ground station) is already under the LEO coverage and established communication with the satellite, represented as line of communication (GS-Sat segment) in Fig. 1.b).

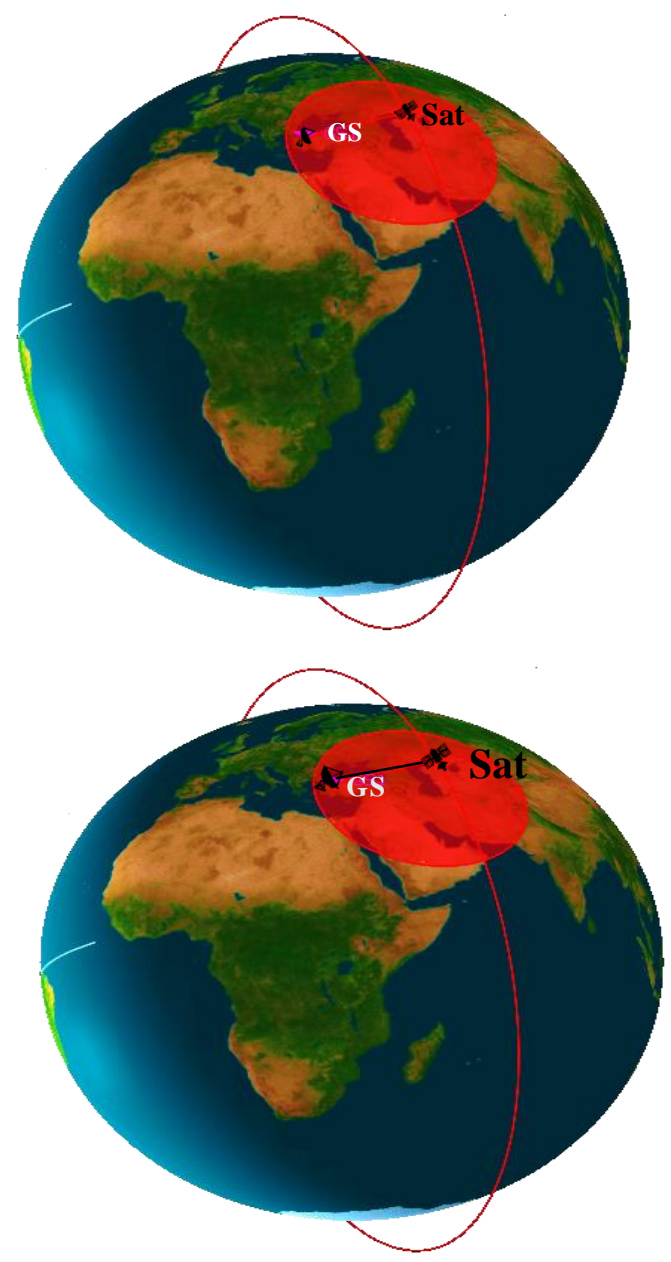

Fig. 1.a) No lock established and b) Lock is established.

Typically for LEO satellites, because of low altitude and consequently the small coverage, under the satellite pass some ground stations may fall under the footprint and some others remain out, which have to wait for another satellite pass to be served. Due to the satellite pass given in Fig. 2, only the ground station identified as $\mathrm{BC} 1$, may establish the communication with the satellite during the appropriate satellite pass.

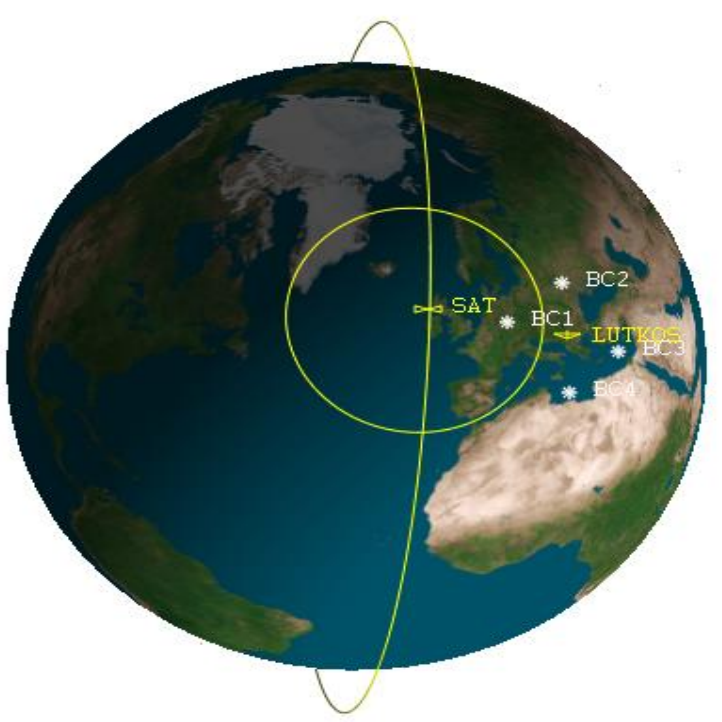

Fig. 2. The satellite coverage area.

On the other side, the position of the satellite within its orbit seen from the ground station is defined by Azimuth $(A z)$ and Elevation $(E l)$ angles. The azimuth is the angle of the direction of the satellite, measured in the ground station's horizon plane from geographical north in clockwise direction (Fig. 3). The range of azimuth is $0^{\circ}$ to $360^{\circ}$. The elevation is the angle between a satellite and the ground station's horizon plane (Fig. 3 ). The range of elevation is $0^{\circ}$ to $90^{\circ}$. The basic geometry between a satellite and ground station is depicted in Fig. 3 [8]. Vectors respectively $\vec{r}, \vec{d}, \overrightarrow{R_{e}}$ represent: the vector from the Earth's center to a satellite, the vector from the ground station to a satellite and the vector from Earth's center to a ground station. This relationship can be clearly shown as:

$$
\vec{d}=\vec{r}-\vec{R}_{e}
$$

The two points of the triangle indicate the satellite and a ground station, and the third one is the Earth's center. Two sides of this triangle are usually known (the distance from the ground station to the Earth's center, what is factically the Earth's radius $R_{e}=6378 \times 10^{3} \mathrm{~m}$, and the distance from the satellite to Earth's center is orbital radius). The most needed parameter is the slant range $d$ (distance from the ground station to the satellite, respectively the communication line length). The 'grey circle' in Fig. 3, represents the ideal horizon plane of the respective ground station, which is always perpendicular to the vector $R_{e}$. Further the triangle from Fig. 3 is brought on plane and is given in Fig. 4. There are four variables in this triangle: $\varepsilon_{0}$ - is elevation angle, $\alpha_{0}$ - is nadir angle, $\beta_{0}{ }^{-}$is central angle and $\boldsymbol{d}$ is slant range. The slant range (communication line length) will be used during link budget calculations, and it is expressed through elevation angle $\varepsilon_{0}$ [9]. 


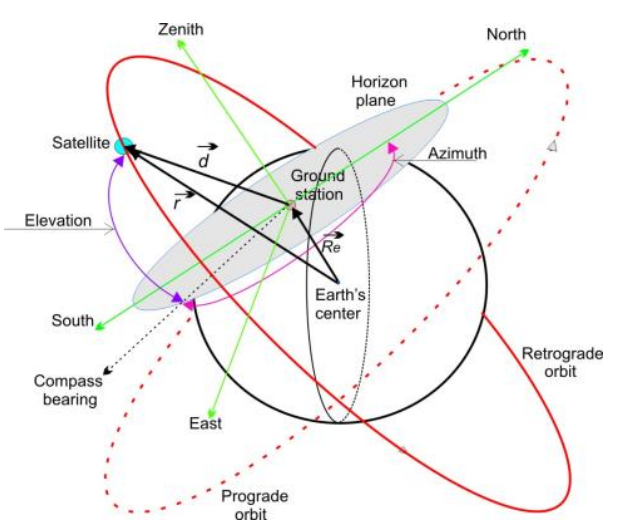

Fig. 3. Horizon plane and orbits.

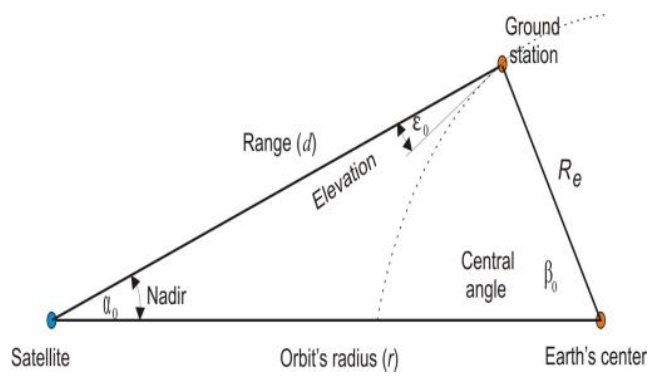

Fig. 4. Ground station geometry.

Solving this triangle yields out the range $d$ as [9]:

$$
d\left(\varepsilon_{0}\right)=R_{e}\left[\sqrt{\left(\frac{H+R_{e}}{R_{e}}\right)^{2}-\cos ^{2} \varepsilon_{0}}-\sin \varepsilon_{0}\right]
$$

$H$ is the LEO satellite orbit altitude. The range $d$ is the communication line length (segment GS-Sat in Fig.1.b).

The shortest range is achieved under $90^{\circ}$ elevations and the largest range occurs at $0^{\circ}$ elevation [10]. The shortest range is equal to the orbital altitude (applying (2) for $90^{\circ}$ ), as:

$$
d\left(\varepsilon_{0}=90\right)=H
$$

The range under the $0^{\circ}$ elevation (applying (2) for $0^{\circ}$ ) is known as the radius of the ideal horizon plane of the appropriate ground station, expressed as:

$$
d\left(\varepsilon_{0}=0\right)=\sqrt{H\left(H+2 R_{e}\right)}
$$

The horizon plan expands as the satellite radius (altitude) increases, presented in Fig. 5 for simulated altitudes from $600 \mathrm{~km}$ to $1200 \mathrm{~km}$ [11].

The range under the lowest elevation angle represents the worst link budget case, since that range is the maximal possible distance between the ground station and the satellite, thus representing the longest communication line (distance).

This range under the same elevation increases as satellite's altitude increases, and based on Eqn. (2) results for different altitudes are given in Table 1 .

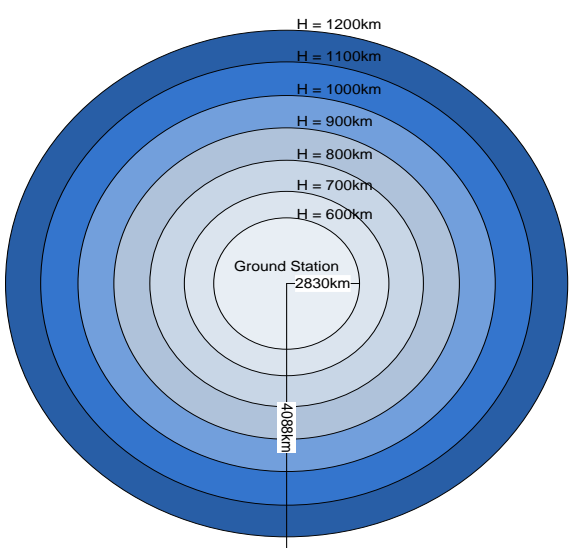

Fig. 5. Ideal horizon plane for different altitudes.

For designed horizon plane, the elevation range of (0-30) ${ }^{\circ}$ is considered. By $0^{\circ}$ is represented ideal horizon plane, and from $5^{\circ}$ to $30^{\circ}$ designed horizon planes. The designed horizon plane is determined by minimal elevation. For simulation purposes the elevation of designed horizon planes is increased in steps by $5^{\circ}$. (In Table 1, the ideal plane is grey shaded to correspond with the respective one at Fig. 3.)

TABLE I

LEO SATELLITE RANGES

\begin{tabular}{|c|c|c|c|c|c|}
\hline \multicolumn{2}{|c|}{$\begin{array}{c}\text { Orbital } \\
\text { Attitude } \\
{[\mathbf{k m}]}\end{array}$} & $\begin{array}{c}\text { H } \\
\mathbf{6 0 0} \\
{[\mathbf{k m}]}\end{array}$ & $\begin{array}{c}\mathbf{H} \\
\mathbf{8 0 0} \\
{[\mathbf{k m}]}\end{array}$ & $\begin{array}{c}\text { H } \\
\mathbf{1 0 0 0} \\
{[\mathbf{k m}]}\end{array}$ & $\begin{array}{c}\text { H } \\
\mathbf{1 2 0 0} \\
{[\mathbf{k m}]}\end{array}$ \\
\hline $\begin{array}{c}\text { Horizon } \\
\text { plane }\end{array}$ & Elevation $\left(\varepsilon_{0}\right)$ & $\begin{array}{c}\text { Range } \\
{[\mathrm{km}]}\end{array}$ & $\begin{array}{c}\text { Range } \\
{[\mathrm{km}]}\end{array}$ & $\begin{array}{c}\text { Range } \\
{[\mathrm{km}]}\end{array}$ & $\begin{array}{c}\text { Range } \\
{[\mathrm{km}]}\end{array}$ \\
\hline Ideal & $0^{\circ}$ & 2830 & 3289 & 3708 & 4088 \\
\hline \hline Designed & $5^{\circ}$ & 2333 & 2784 & 3197 & 3574 \\
\hline Designed & $10^{\circ}$ & 1942 & 2372 & 2770 & 3136 \\
\hline Designed & $15^{\circ}$ & 1663 & 2035 & 2414 & 2764 \\
\hline Designed & $20^{\circ}$ & 1386 & 1765 & 2120 & 2453 \\
\hline Designed & $25^{\circ}$ & 1219 & 1563 & 1895 & 2208 \\
\hline Designed & $35^{\circ}$ & 1070 & 1392 & 1701 & 1996 \\
\hline
\end{tabular}

Tracking satellite software, based on Kepler's elements, precisely define relative position between the ground station and the satellite, consequently determining also the time when the satellite will lock/unlock communication with the ground station. This software provides real-time tracking information, usually displayed in different modes (satellite view, radar map, tabulated, etc). The display mode "radar map" includes accurate satellite position with the ground station considered at the center, as in Figure 6 is presented $[10,11]$.

The perimeter of the circle is the horizon plane (grey circle from Figure 2), with North on the top $\left(A z=0^{\circ}\right)$, then East $(A z$ $\left.=90^{\circ}\right)$, South $\left(A z=180^{\circ}\right)$ and West $\left(A z=270^{\circ}\right)$. Three concentric circles represent different elevations: $0^{\circ}, 30^{\circ}$ and $60^{\circ}$. At the center the elevation is $\varepsilon_{0}=90^{\circ}$. Most usual software parameters which define the movement of the satellite related to the ground station are: $A O S_{\text {time }}-$ Acquisition of the satellite (time), $\operatorname{LOS}_{\text {time }}$ - Loss of the satellite (time), $A O S_{A z}$-Acquisition of the satellite (azimuth), $\operatorname{LOS}_{A z}-$ Loss of the satellite (azimuth), Max El-Maximal Elevation [9]. 


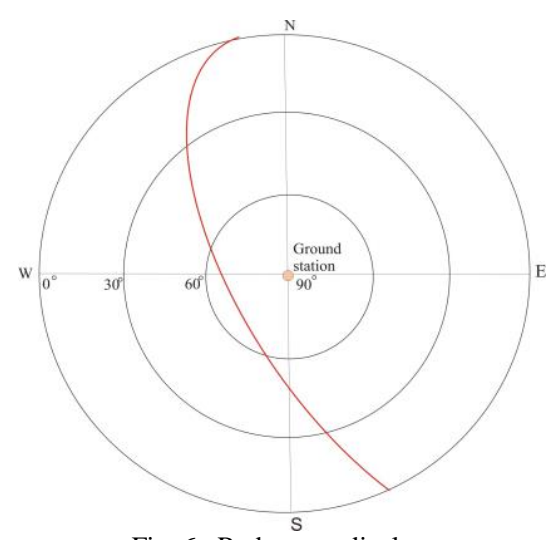

Fig. 6. Radar map display.

The satellite's movement (satellite's pass) is presented with the satellite's path in a radar map (red curve) what is in fact the satellite's orbit projection on the ideal horizon plane (Figure 3). Because of Earth's rotation around its N-S axis the ground station changes the position relatively to orbital plane, so the pointing (azimuth and elevation) from the ground station to the satellite is not identical for different satellite passes, hence the communication duration between the satellite and the appropriate ground station differs under different satellite passes, presented in Fig. 6.

\section{Designed Ground Station Horizon Plane}

The outer circle at $0^{\circ}$ elevation at radar map display at Figure 6 represents the ideal horizon plane. The ideal communication duration between the satellite and the ground station is defined by instant of the acquisition and the loss of satellite under $0^{\circ}$ elevation. The communication duration is the difference of the satellite's acquisition and loss time, as:

$$
\text { Communication. Duration }=A O S_{\text {time }}-L O S_{\text {time }}
$$

But, practically the acquisition and loss of satellite do not occur at $0^{\circ}$ elevation, because of natural barriers or too high buildings within area of the ideal horizon plane [11]. Further the case depicted in Figure 7 is analyzed. Theoretically, based on Kepler's laws for the case in Figure 7 the communication between the satellite and the ground station should be established at point $\mathrm{A}\left(A z \approx 19^{\circ}\right.$ under $0^{\circ}$ elevation $)$ and communication should be lost at point $\mathrm{B}\left(A z \approx 178^{\circ}\right.$ under $0^{\circ}$ elevation). But, no contact with the satellite can be established under a $0^{\circ}$ elevation, because of natural barriers.

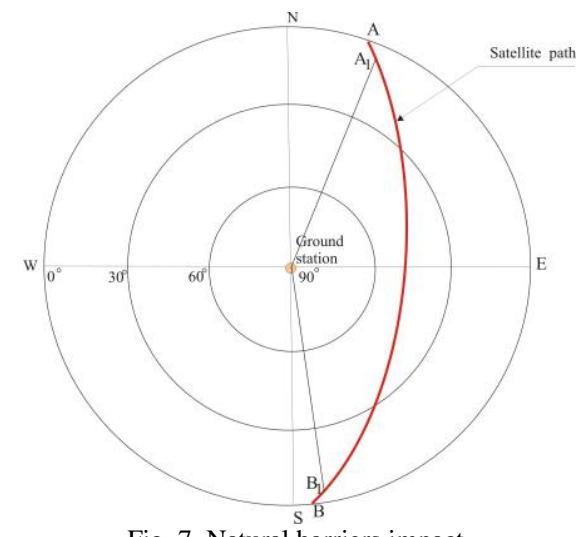

Fig. 7. Natural barriers impact.
Practical contact between the satellite and the ground station is established at point $A_{1}$ and lost at point $B_{1}$ (both under elevation of few degrees). Thus, points $A$ and $B$ belong to the ideal horizon plane, while $A_{1}$ and $B_{1}$ belong to the practical horizon (practical lock/unlock). If it is considered the whole horizon in the azimuth range of $0^{\circ}-360^{\circ}$, in any direction of the horizon plane the natural barriers will differ; consequently so will the acquisition and loss elevation (points $A_{1}$ and $B_{1}$ ). Different points for acquisition and loss, for different azimuths creates the broken circle (black one). The broken circle represents the practical horizon impacted by natural barriers. Practical horizon it is not a flat plane. Each edge point of the broken circle is the practical elevation of acquisition or the loss of the satellite. Usually the lock is established and lost in average at elevation angles of $1^{\circ}-4^{\circ}$ [10]. This is presented in Figure 8 [11, 12]. For the same satellite orbit, the distances between the satellite and the ground station, will differ for different satellite passes. This difference in distances implies different power from the satellite to be transmitted toward the ground station in order to keep a similar performance (signal to noise ratio). The lowest power is required at $90^{\circ}$ elevation and the highest power is required when the satellite is at $0^{\circ}$ elevation, consequently representing the worst propagation case for the link budget. Thus, the implementation of a designed horizon plane, in one hand avoids the impact of obstacles caused by the natural barriers and on other hand applies the minimal power under the worst propagation case for the link budget calculations.

The designed horizon plane is defined by the minimal elevation considered for the acquisition and the loss of satellite, based on records done at the site (known as ground station mask), where the ground station is going to be established. For different purposes of the satellite systems, the minimal elevation value for the designed horizon plane ranges on $(5-30)^{\circ}$, what is considered at Table 1 (For example, the NOAA's search and rescue ground stations apply $5^{\circ}$ for the designed horizon plane [12]).

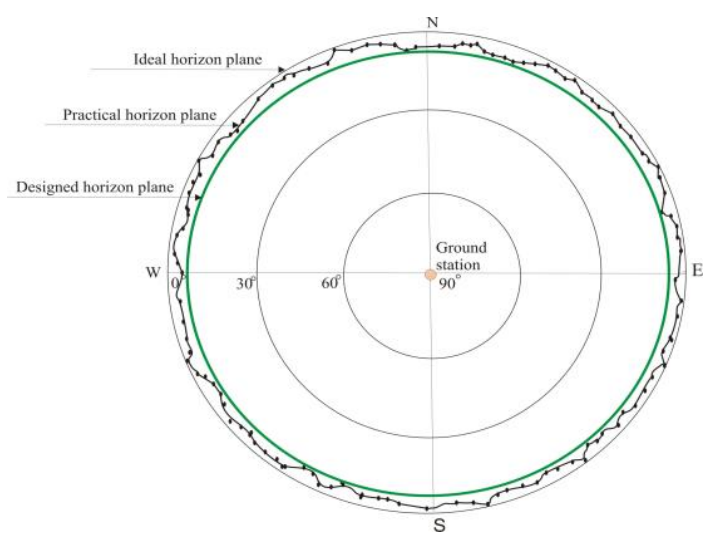

Fig. 8. Ideal, practical, and designed horizon plane.

In Figure 9 the LEO satellite is seen from the ground station six times during the day (86400s), where the longest communication is achieved due to the fifth pass under elevation of $45^{\circ}$ and the shortest communication due to the forth pass under elevation of $5^{\circ}$. 
Mathematically, these planes are expressed by the range from the ground station to the satellite (circle's radius), as follows: For the ideal horizon plane, the radius $d_{I}$ is:

$$
d_{I}\left(\varepsilon_{0}=0\right)=\sqrt{H\left(H+2 R_{e}\right)}
$$

For the designed horizon plane the radius $d_{D}$ is:

$$
d_{D}\left(\varepsilon_{0}=X\right)=R_{e}\left[\sqrt{\left(\frac{H+R_{e}}{R_{e}}\right)^{2}-\cos ^{2} X}-\sin X\right]
$$

where $X$ is minimal determined elevation for designed horizon plane.

For any point of practical horizon with elevation of $x$, the distance $d_{P}$ is:

$$
d_{P}\left(0<\varepsilon_{0}=x<X\right)=R_{e}\left[\sqrt{\left(\frac{H+R_{e}}{R_{e}}\right)^{2}-\cos ^{2} x}-\sin x\right]
$$

For the case under $90^{\circ}$ elevation, the distance is fixed and denoted as $d_{H}$ (the lowest possible range) expressed as:

$$
d_{H}\left(\varepsilon_{0}=90\right)=H
$$

The mathematical correlation of practical horizon related to ideal and designed horizon planes is expressed as follows:

$$
\begin{aligned}
& \lim _{\varepsilon_{0}=x \rightarrow 0} d_{P}\left(0<\varepsilon_{0}=x<X\right)=d_{I} \\
& \lim _{\varepsilon_{0}=x \rightarrow X} d_{P}\left(0<\varepsilon_{0}=x<X\right)=d_{D}
\end{aligned}
$$

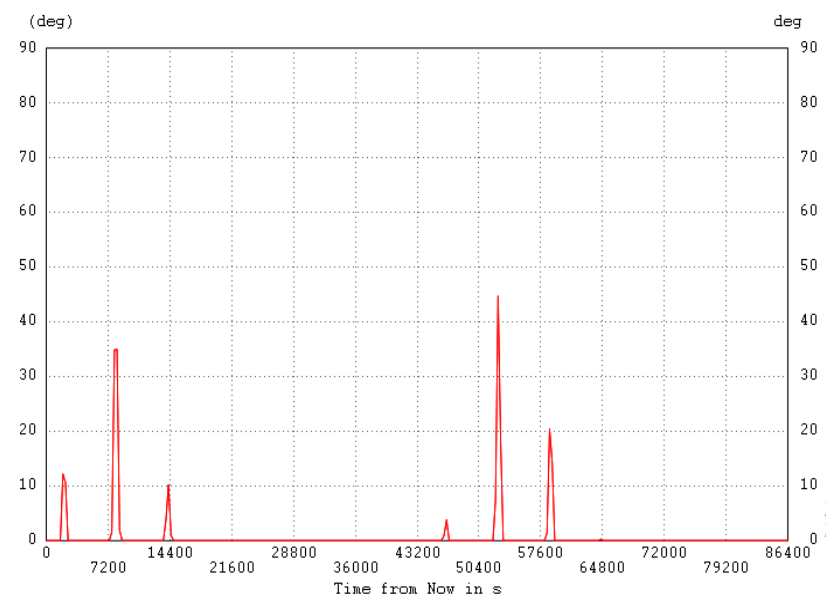

Fig. 9. Communication duration between the satellite and the ground station (simulation).

These two equations (Eqn. 9, Eqn. 10) confirm that any point of the practical horizon lies within a ring made by designed and ideal horizon planes (Figure 8).

Further, this paper discusses the savings on transmitted power from the satellite toward the ground station, by implementation of designed horizon plane compared to the case of ideal horizon plane and practical horizon.

\section{DOWNLINK BUDGET}

For downlink budget calculations, of the greatest interest is the receiving system signal to noise ratio $\left[(S / N)\right.$ or $\left.\left(S / N_{0}\right)\right]$ expressed by range equation [13-15], as:

$$
\frac{S}{N_{0}}=\frac{\operatorname{EIRP}\left(G / T_{S}\right)}{k L_{S} L_{0}}
$$

where EIRP is Effectively Isotropic Radiated Power from the satellite transmitter. Considering that $N=N_{0} B, N_{0}=k T$ where, $N_{0}$ is spectral noise density, $B$ ground station receiver bandwidth, $k=1.38 \cdot 10^{-23} \mathrm{~W} / \mathrm{HzK}$ is Boltzmann's constant yields out:

$$
(S / N)=\left(S / N_{0}\right)-B
$$

Expressing (11) in decibels yields:

$$
\frac{S}{N_{0}}(d B / H z)=E I R P-L_{S}-L_{0}+G / T_{S}+228.6
$$

$L_{S}$ is free space loss, $L_{0}$ denotes other losses (atmospheric loss, polarization loss, misspointing etc) and $G / T_{S}$ is Figure of merit. The reception quality of the satellite receiving system is commonly defined through a Receiving System Figure of Merit as $G / T_{S}[10,14]$ :

$$
T_{S}=T_{A}+T_{\text {comp }}
$$

where $G$ is receiving antenna gain, $T_{S}$ is receiving system noise temperature, $T_{A}$ is antenna noise temperature and $T_{\text {comp }}$ is composite noise temperature of the receiving system, including lines and equipment [10,14].

The Figure of Merit $G / T_{S}$ expresses the impact of external and internal noise factors. Free space loss $\left(L_{S}\right)$ is the greatest loss in transmitted power due to the long distance between the satellite and a ground station. This loss is displayed as [14]:

$$
L_{S}=\frac{(4 \pi d)^{2}}{\lambda^{2}}
$$

where $d$ is the distance (slant range) between the satellite and a ground station, and $\lambda$ is the signal wavelength. The free space loss $L_{S}$ is often convenient to be expressed as function of distance $d$ and signal frequency $f$, and then $L_{S}$ is [12],

$$
L_{S}\left(\varepsilon_{0}\right)=\left(\frac{4 \pi f}{c}\right)^{2} \cdot d^{2}\left(\varepsilon_{0}\right)
$$

where $d\left(\varepsilon_{0}\right)$ is represented by (2). Free space loss increases by both, frequency and the distance.

The downlink margin (DM) is defined as:

$$
D M=(S / N)_{r}-(S / N)_{r q d}
$$

where the $r$ indicates expected signal to noise ratio to be received at receiver, and $r q d$ means required signal to noise ratio requested by customer, as in advance defined performance criteria. The positive value of $D M$ is indicates that the link is closed (communication is established). 


\section{POWER SAVING SIMULATION}

The further simulation elaborates the EIRP savings by implementing the designed horizon plane instead of ideal one, due to keeping constant downlink receiving margin. For simulation purposes are considered altitudes of $600 \mathrm{~km}$, $800 \mathrm{~km}, 1000 \mathrm{~km}$ and $1200 \mathrm{~km}$ as typical LEO altitudes. For these altitudes applying (2) it is calculated the range from a hypothetical ground station to the satellite at appropriate altitudes, and presented at Table 1 . Table 1 gives ranges for ideal horizon plane at $0^{\circ}$, and designed horizon plane starting at $5^{\circ}$ up to $30^{\circ}$. The designed horizon plane is changed by steps of $5^{\circ}$.

Table 1. shows that the distance (slant range) between the LEO satellite and the ground station changes and depends on elevation angle. This causes different free space loss and consequently different signal to noise ratio (see Eqn. (13)) and different downlink margin, respectively.

The idea behind this paper is the assumption of keeping the constant downlink margin over different distances between the LEO satellite and the appropriate ground station. Thus,

$$
D M=(S / N)_{r}-(S / N)_{\text {rqd }}=\text { constant }
$$

Considering that the required $(S / N)$ level is in advance determined by user requirements, yields out that must be:

$$
(S / N)_{r}=\text { constant }
$$

and since it is

$$
(S / N)_{r}=\left(S / N_{0}\right)_{r}-B
$$

where $B$ is determined receiving system bandwidth, and the simulation is applied for the ground system with already determined bandwidth, yields out that should be:

$$
\left(S / N_{0}\right)_{r}=\text { constant }
$$

The LEO satellite path over the ground station is characterized by two typical events (extreme cases), the first one, when the satellite is seen due to the longest distance from the ground station (for ideal horizon plane under $0^{\circ}$ elevation, or for designed horizon plane under $X^{\mathrm{o}}$ elevation), and the second one, when the satellite is seen perpendicularly from the ground station (under $90^{\circ}$ elevation). To fulfill the condition expressed by (21), the lowest EIRP is required under $90^{\circ}$ elevation and the highest EIRP is required for ideal horizon plane under $0^{\circ}$, or for designed horizon plane under $X^{\circ}$. The lowest required EIRP, for the both, ideal or designed horizon plane, is the same since in both cases the range is $H$ (satellite's altitude).

Under elevation $x^{0}$ and neglecting other loss the range equation (13) becomes as:

$$
\left(\frac{S}{N_{0}}\right)_{\left(\varepsilon_{0}=x\right)}=\operatorname{EIRP}_{\left(\varepsilon_{0}=x\right)}-L_{S\left(\varepsilon_{0}=x\right)}+\left(\frac{G}{T_{S}}\right)+228.6
$$

$\left(G / T_{S}\right)$ it is not indexed by elevation, since the environmental factors and technical equipment parameters do not depend on elevation (See Eqn. (14)). Then, (21) becomes:
$\left(\frac{S}{N_{0}}\right)_{\left(\varepsilon_{0}=0\right)}=\left(\frac{S}{N_{0}}\right)_{\left(\varepsilon_{0}=x\right)}=\cdots=\left(\frac{S}{N_{0}}\right)_{\left(\varepsilon_{0}=X\right)}=\left(\frac{S}{N_{0}}\right)_{\left(\varepsilon_{0}=90\right)}$

Being aware that the elevations of $0, x, X$, and 90 refer respectively to ideal horizon plane $(I)$, practical horizon $(P)$, designed horizon plane $(D)$ and to the perpendicular distance $(H)$, the Eqn. (23) can be rewritten as:

$$
\left(\frac{S}{N_{0}}\right)_{I}=\left(\frac{S}{N_{0}}\right)_{P}=\left(\frac{S}{N_{0}}\right)_{D}=\left(\frac{S}{N_{0}}\right)_{H}
$$

Applying (22) will have following four equations:

$$
\begin{aligned}
& \left(\frac{S}{N_{0}}\right)_{I}=E I R P_{I}-L_{S(I)}+\left(\frac{G}{T_{S}}\right)+228.6 \\
& \left(\frac{S}{N_{0}}\right)_{P}=E I R P_{I}-L_{S(P)}+\left(\frac{G}{T_{S}}\right)+228.6 \\
& \left(\frac{S}{N_{0}}\right)_{D}=E I R P_{D}-L_{S(D)}+\left(\frac{G}{T_{S}}\right)+228.6 \\
& \left(\frac{S}{N_{0}}\right)_{H}=E I R P_{H}-L_{S(H)}+\left(\frac{G}{T_{S}}\right)+228.6
\end{aligned}
$$

For the communication between the satellite and the ground station, the EIRP transmitted from the satellite toward the station should vary respectively as follows, for ideal horizon plane, for designed horizon plane and practical horizon.

$$
\begin{gathered}
\text { From }_{E I R P_{H}} \text { to } \operatorname{EIRP}_{I} \\
\text { From } \operatorname{EIRP}_{H} \text { to } \operatorname{EIRP}_{D} \\
\text { From } \operatorname{EIRP}_{H} \text { to } \operatorname{EIRP}_{P} \\
\text { EIRP }_{H}<\operatorname{EIRP}_{D}<\operatorname{EIRP}_{P}<\operatorname{EIRP}_{I}
\end{gathered}
$$

Applying (24) yields out as:

$$
E I R P_{I}-L_{S(l)}=E I R P_{P}-L_{S(P)}=E I R P_{D}-L_{S(D)}=E I R P_{H}-L_{S(H)}(27)
$$

Above Eqn. tells us that larger free space loss (larger distance) more power is needed to be radiated from the satellite in order to keep very constant performance.

Since this paper is concerned about the comparison on the power saving under the circumstances of ideal and designed horizon plane, further is considered:

For:

$$
E I R P_{I}-L_{S(I)}=E I R P_{D}-L_{S(D)}
$$

$$
\begin{gathered}
\Delta E I R P=E I R P_{I}-E I R P_{D} \\
\Delta E I R P=L_{S(I)}-L_{S(D)}
\end{gathered}
$$

Finally, applying (16) for the case of the ideal horizon (0 elevation) and for the designed horizon plane ( $X$ elevation), than the saving on EIRP for the designed horizon plane considered under the minimal elevation of $X$, compared to the power needed for the communication at circumstances of ideal horizon plane ( 0 elevations) is expressed as: 


$$
\Delta E I R P_{X}(d B)=20 \log \frac{d\left(\varepsilon_{0}=0\right)}{d\left(\varepsilon_{0}=X\right)}
$$

Applying distances from Table 1 at Eqn. (31), results in Table 2 represent the EIRP savings for designed horizon planes determined by different minimal elevation compared to ideal horizon plane. These results are also given in Figure 10.

TABLE II

POWER SAVINGS (AEIRP)

\begin{tabular}{|c|c|c|c|c|c|}
\hline \multicolumn{2}{|c|}{$\begin{array}{c}\text { Orbital } \\
\text { Attitude } \\
{[\mathbf{k m}]}\end{array}$} & $\begin{array}{c}\mathbf{H} \\
\mathbf{6 0 0} \\
{[\mathbf{k m}]}\end{array}$ & $\begin{array}{c}\mathbf{H} \\
\mathbf{8 0 0} \\
{[\mathbf{k m}]}\end{array}$ & $\begin{array}{c}\mathbf{H} \\
\mathbf{1 0 0 0} \\
{[\mathbf{k m}]}\end{array}$ & $\begin{array}{c}\mathbf{H} \\
\mathbf{1 2 0 0} \\
{[\mathbf{k m}]}\end{array}$ \\
\hline $\begin{array}{c}\text { Horizon } \\
\text { plane }\end{array}$ & $\begin{array}{c}\text { Elevation } \\
\left(\varepsilon_{0}\right)\end{array}$ & $\begin{array}{c}\Delta \mathrm{EIRP} \\
{[\mathrm{dB}]}\end{array}$ & $\begin{array}{c}\Delta \mathrm{EIRP} \\
{[\mathrm{dB}]}\end{array}$ & $\begin{array}{c}\Delta \mathrm{EIRP} \\
{[\mathrm{dB}]}\end{array}$ & $\begin{array}{c}\Delta \mathrm{EIRP} \\
{[\mathrm{dB}]}\end{array}$ \\
\hline \hline Designed & $5^{\circ}$ & 1.67 & 1.44 & 1.28 & 1.16 \\
\hline Designed & $10^{\circ}$ & 3.27 & 2.85 & 2.53 & 2.30 \\
\hline Designed & $15^{\circ}$ & 4.61 & 4.16 & 3.73 & 3.39 \\
\hline Designed & $20^{\circ}$ & 6.20 & 5.40 & 4.85 & 4.43 \\
\hline Designed & $25^{\circ}$ & 7.31 & 6.46 & 5.83 & 5.35 \\
\hline Designed & $30^{\circ}$ & 8.44 & 7.47 & 6.77 & 6.22 \\
\hline
\end{tabular}

From Table 2, it is obvious that for altitude of $800 \mathrm{Km}$, under elevation of $10^{\circ}$, the savings on EIRP are almost $3 \mathrm{~dB}$ $(2.85 \mathrm{~dB})$, what means that by implementation of designed horizon plane the power transmitted from the satellite toward the ground station may be approximately a half of the power needed under the circumstances of the ideal horizon plane.

Figure 10 shows that for in advance determined satellite altitude $(H)$, the power savings due to the implementation of designed horizon plane, increases as minimal elevation increases. On the right angle of the Figure 10, X denotes minimal elevation for designed horizon plane. In Figure 10, for the altitude of $800 \mathrm{Km}$, under elevation of $10^{\circ}$, the power saving is lined as a second brick from the bottom (brown colour) identifying exactly the savings of $(2.85 \mathrm{~dB})$. For the altitude of $800 \mathrm{Km}$, "playing" with elevation on the range of $(5-30)^{\circ}$ the length of bricks vary from (1.44 to 7.47$) \mathrm{dB}$, in fact representing the range of power savings. The similar applies for other altitudes under different elevations.

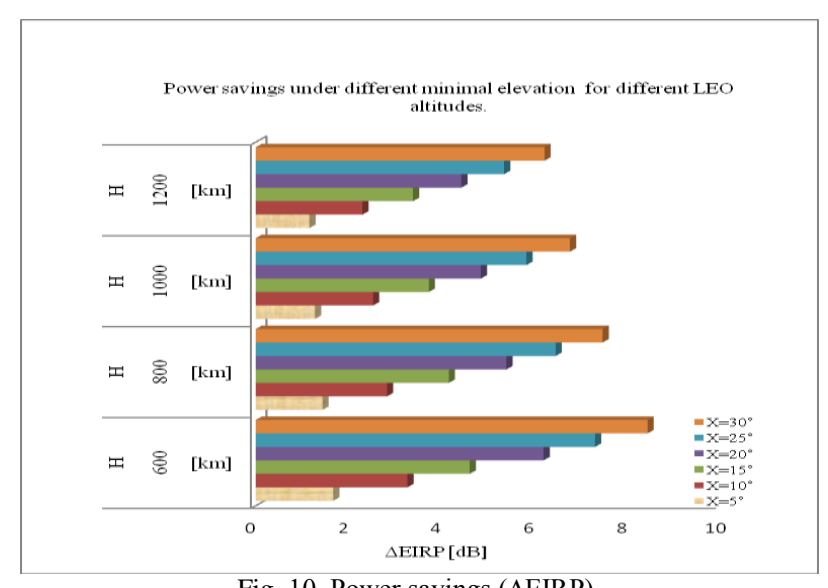

Fig. 10. Power savings ( $\triangle E I R P)$.
Further, it is elaborated, how to calculate the minimal elevation for the designed horizon plane, for an in advance determinated requirement related to the EIRP savings from the satellite toward the ground station. Thus further goal is to calculate $X$, for in advanced required $\triangle E I R P$. Thus the minimal elevation angle is required to be calculated. Eqn. (31) may be rewritten as:

$$
d\left(\varepsilon_{0}=X\right)=\frac{d\left(\varepsilon_{0}=0\right)}{10^{\frac{\Delta E I R P}{20}}}
$$

If Eqn. (32) is equalized with (6) and making substitutes as,

$$
\begin{gathered}
A=\frac{d\left(\varepsilon_{0}=0\right)}{10^{\frac{\Delta E I R P}{20}}}=\frac{\sqrt{H\left(H+R_{e}\right)}}{10^{\frac{\Delta E I R P}{20}}} \\
B=\left(\frac{H+R_{e}}{R_{e}}\right)^{2}
\end{gathered}
$$

stems out, the following equation:

$$
\left(\frac{A}{R_{e}}\right)^{2}+2\left(\frac{A}{R_{e}}\right) \sin X-(B-1)=0
$$

and the final solution is:

$$
\sin X=\frac{B-\left[1+\left(\frac{A}{R_{e}}\right)^{2}\right]}{2\left(\frac{A}{R_{e}}\right)}
$$

For a satellite orbit of $H$ altitude, can be implemented a satellite ground station with designed horizon plane of minimal elevation $X$ what will save a $\triangle E I R P$ compared to the EIRP needed for the ideal horizon plane of the same station. Under circumstances of saving a half of EIRP, this means savings of $3 \mathrm{~dB}$, for different altitudes it is calculated the minimal elevation window, and given in Table 3.

TABLE III

ELEVATION WINDOW

\begin{tabular}{|c|c|c|c|c|}
\hline Orbital Attitude $[\mathbf{k m}]$ & $\begin{array}{c}\mathbf{H} \\
\mathbf{6 0 0} \\
{[\mathbf{k m}]}\end{array}$ & $\begin{array}{c}\mathbf{H} \\
\mathbf{8 0 0} \\
{[\mathbf{k m}]}\end{array}$ & $\begin{array}{c}\mathbf{H} \\
\mathbf{1 0 0 0} \\
{[\mathbf{k m}]}\end{array}$ & $\begin{array}{c}\mathbf{H} \\
\mathbf{1 2 0 0} \\
{[\mathbf{k m}]}\end{array}$ \\
\hline Designed horizon plane & $\begin{array}{c}\Delta \mathrm{EIRP}= \\
3[\mathrm{~dB}]\end{array}$ & $\begin{array}{c}\Delta \mathrm{EIRP}= \\
3[\mathrm{~dB}]\end{array}$ & $\begin{array}{c}\Delta \mathrm{EIRP} \\
= \\
3[\mathrm{~dB}]\end{array}$ & $\begin{array}{c}\Delta \mathrm{EIRP}= \\
3[\mathrm{~dB}]\end{array}$ \\
\hline \hline $\begin{array}{c}\text { Estimated minimal } \\
\text { elevation }\end{array}$ & $9.2^{\circ}$ & $10.5^{\circ}$ & $11.9^{\circ}$ & $12.9^{\circ}$ \\
\hline
\end{tabular}

Finally, the elevation window for designed horizon plane, for LEO altitudes, in order to be transmitted only a half of EIRP compared to the case of ideal horizon plane, ranges from $9.2^{\circ}$ to $12.9^{\circ}$. Table 3 , shows that for the satellite at altitude of $800 \mathrm{Km}$, to communicate toward the ground station with the 
designed horizon plane at elevation of $10.5^{\circ}$, half of EIRP will be needed for the safe communication compared to the case under ideal horizon plane at the same ground station. The same applies for other altitudes.

\section{CONCLUSION}

The paper presents an analytical approach about designed horizon plane determination related to the ideal one. The implementation of a designed horizon plane, in one hand avoids the impact of obstacles caused by the natural barriers and on other hand applies the minimal power under the worst propagation case for the link budget calculations. The paper provides methodology on designed horizon plane implementation and advantages compared to an ideal horizon plane. Calculations about power savings to be transmitted from the satellite are given, but also the determination of minimal elevation for the designed horizon plane for an in advance power requirements are provided. The elevation window for designed horizon plane, for LEO altitudes, in order to be transmitted only a half of EIRP compared to the case of ideal horizon plane, ranges from $9.2^{\circ}$ to $12.9^{\circ}$.

\section{REFERENCES}

[1] A. Botta, A. Pescape, "New generation satellite broadband Internet service: should ADSL and 3G worry", TMA 2013, co-lacted with IEEE INFOCOM 2013, April 2013, Turin, Italy, pp.3279-3284. DOI: $10.1109 /$ INFCOM.2013.6567151.

[2] P. Garner, D. Cooke and A. Haslehurst, "Development of a scalable Payload Downlink chain for highly agile earth observation missions in Low Earth Orbit" $4^{\text {th }}$ International Conference Recent Advances in Space Technologies, Isntanbul, Turkey, 2009, pp.529-534, DOI: 10.1109/RAST.2009.5158251.

[3] Tarun S. Tuli, Nathan G. Orr, Dr. Robert E. Zee, "Low Cost Ground Station Design for Nanosatellite Missions", North American Space Symposium, AMSAT 2006.

[4] Thomas Choi, Terry H. Stevenson, and E. Glenn Lightsey, "Reference Ground Station Design for University Satellite Missions with Varying Communication Requirements", American Institute of Aeronautics and Astronautics, 55th AIAA Aerospace Sciences Meeting 9 - 13 January 2017, Grapevine, Texas, AIAA 1334

[5] L. Acquaroli and B. Morelli,"Agile payload development and testing of the simplified qualification model" Proceedings of the European Conference on Spacecraft Structures, Materials and Mechanical Testing, 10-12 May 2005, Noordwijk, The Netherlands 2005, Bibliographic Code: 2005ESASP.581E..56A.

[6] Samir Medina Perlaza, Evelio Astaiza Hoyos and Pedro Vera Vera. "Reconfigurable Satellite Payload Model based on Software Radio Technologies" 3rd IEEE International Congress of the Andean Region, November 2006, Ecuador, ANDESCON2006, 1-6,

[7] B. Kamo, S. Cakaj, E. Agastra, "Downlink Free Space Loss Compensation through Receiver Bandwidth Selectivity at LEO Satellite Ground Stations", IEEE SCVT 2016, $23^{\text {rd }}$ Symposium on Communications and Vehicular Technology in the Benelux, Mons, Belgium, 22 nov. 2016, pp. 1-5, DOI: 10.1109/SCVT.2016.7797671.

[8] S. Cakaj, "Practical Horizon Plane and Communication Duration for Low Earth Orbiting (LEO) Satellite Ground Stations", WSEAS Journal: Transactions on Communications, Issue 4, Volume 8, April 2009, pp. 373-383

[9] G.D. Gordon, W.L. Morgan, Principles of communication satellites, John Wiley \& sons, Inc. 1993, ISBN-13: 978-0471557968.

[10] S. Cakaj, K. Malaric, "Rigorous analysis on performance of LEO satellite ground station in urban environment", International Journal of Satellite Communications and Networking, 25 (2007), 6; pp. 619-643 (Journal article), London, UK, doi/abs/10.1002/sat.895.

[11] S. Cakaj, B. Kamo, V. Kolici, O. Shurdi, "The Range and Horizon Plane Simulation for Ground Stations of Low Earth Orbiting (LEO) Satellites", International Journal of Communications, Networks and
System Sciences (IJCNS), Volume 4, Number 9, September 2011, USA, pp. 585-589, DOI: 104236/ijcns.2011.49070.

[12] S. Cakaj, "Local User Terminals for Search and Rescue Satellites", Book, VDM Publishing House, Saarbrucken, Germany, pages 84, September 2010, ISBN-13: 978-3639294804.

[13] S. Cakaj, B. Kamo, A. Lala, A. Rakipi, "Elevation Impact on Signal to Spectral Noise Density Ratio for Low Earth Orbiting Satellite Ground Station at S-Band", IEEE Science and Information Conference, 27-29 August, London UK, 2014, pp.641-645, DOI: 10.1109/SAI.2014.6918255.

[14] M. Richharia, "Satellite communication systems", McGraw Hill, New York, 1998, ISBN 13: 9780071342087.

[15] B. Sklar, "Digital Communication", Prentice Hall PTR, New Jersey 2005, ISBN-13: 978-0130847881.

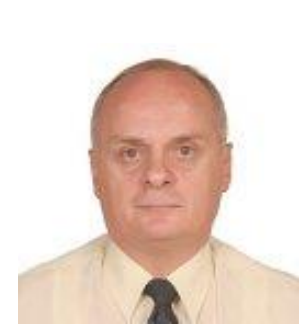

Dr. Shkelzen Cakaj has received his BSc and MSc degrees from Prishtina University in Kosovo. Since 2003 is cooperating with Technical University in Vienna, where he has prepared his Master Thesis. He was awarded a $\mathrm{PhD}$ in area of satellite communications from Zagreb University, in January 2008. Later on, in 2009, as Fulbright scholar, he continued his post doctorate research at NOAA (National Oceanic and Atmospheric Administration) at Maryland, USA. He is the author of 60 papers published in worldwide conferences and journals; mostly IEEE and also engaged as a reviewer. His area of interest is the satellite ground station performance. He is lecturing "Satellite Systems" at Polytechnic University of Tirana (UPT), Albania and "Systems and Signals" at University of Business and Technology (UBT), Prishtina, Kosovo. On 2016 he was awarded as "Albanian ICT Researcher of the Year 2015".

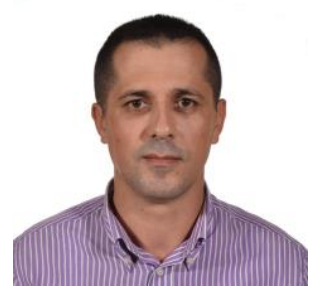

Dr. Bexhet Kamo has received his BSc and MSc degrees in Electronic Engineering, in 1999, from Faculty of Information Technology (FIT) of the Polytechnic University of Tirana, (PUT). In 2008 finished the doctoral studies and awarded "Doctor of Science" at PUT, FIT, where in 2011, he received the academic title "Associate Professor". Since 1999 is working as a pedagogue in PUT, FIT and currently is staff member of this faculty lecturing subjects, "Theory of Signals" and "Systems in Radiofrequency 2". He has about 40 articles and about 20 publications in scientific conferences/journals in the fields related to the Information and Communication Technology. 